\title{
O papel terapêutico do Programa Farmácia Viva e das plantas medicinais no centro-sul piauiense
}

\begin{abstract}
PEREIRA, J.B.A.'; RODRIGUES, M.M.1; MORAIS, I.R.'; VIEIRA, C.R.S.2; SAMPAIO, J.P.M.2; MOURA, M.G.1; DAMASCENO, M.F.M.'; SILVA, J.N. ${ }^{3}$; CALOU, I.B.F.3; DEUS, F.A.4; PERON, A.P.'; ABREU, M.C.'; MILITÃO, G.C.G. ${ }^{5 ;}$ FERREIRA, P.M.P. ${ }^{3,6}$ *

${ }^{1}$ Departamento de Ciências Biológicas, Universidade Federal do Piauí, Rua Cícero Duarte, 905, CEP: 64.607670, Picos-PI, Brasil; ' 2Departamento de Nutrição, Universidade Federal do Piauí, Rua Cícero Duarte, 905, CEP: 64.607-670, Picos-PI, Brasil; ${ }^{3}$ Programa de Pós-Graduação em Ciências Farmacêuticas (PPGCF), Universidade Federal do Piauí, Avenida Universitária, lado ímpar, CEP: 64049-550, Teresina-PI, Brasil; “Laboratório Fitoterápico de Picos (LAFIPI), Programa Farmácia Viva, Avenida Nossa Senhora de Fátima, 550, CEP: 64.600-138, PicosPI, Brasil; ${ }^{5}$ Departamento de Fisiologia e Farmacologia, Universidade Federal de Pernambuco, Rua Nelson Chaves, s/n, CEP: 50670-901, Recife-PE, Brasil; ' ${ }^{\circ}$ Departamento de Biofísica e Fisiologia, Universidade Federal do Piauí, Avenida Universitária, lado ímpar, CEP: 64049-550, Teresina-PI, Brasil. *Autor para correspondência: pmpf@ufpi.edu.br
\end{abstract}

\begin{abstract}
RESUMO: Este trabalho realizou um levantamento sobre o uso de plantas medicinais na cidade de Picos-PI, identificou as plantas cultivadas no horto pertencente ao Laboratório Fitoterápico de Picos (LAFIPI), e analisou o uso de fitoterápicos dispensados pelo Programa Farmácia Viva no triênio 2008-2010. Do total dos 750 entrevistados, 37,6\% foram homens e $62,4 \%$ mulheres, dentre os quais a maioria não concluiu o segundo grau $(69,2 \%)$ e $77,2 \%$ possuíam renda mensal de até dois salários mínimos. Com relação ao consumo de plantas medicinais, $76,3 \%$ afirmaram utilizá-las para tratar doenças, principalmente por considerá-las mais saudáveis $(84,8 \%)$. A indicação do uso foi orientada, sobretudo, por familiares $(82,2 \%)$, embora a maioria adquira as plantas em feiras livres (32,8\%). Das 127 plantas relatadas, as mais citadas foram erva-cidreira, boldo e hortelã, sendo as folhas a parte mais utilizada $(42,3 \%)$, predominantemente por infusão $(39,4 \%)$. As aplicações mais lembradas foram para tratar dores em geral (17\%), distúrbios respiratórios (16,5\%) e digestivos (16\%). As espécies mais cultivadas no horto são chambá (Justicia pectoralis), alecrim pimenta (Lippia sidoides), malva santa (Plectranthus barbatus) e erva cidreira (Lippia alba). O lambedor de chambá foi o fitoterápico mais procurado pela população entre 2008 e 2010. Esse estudo descreveu, pela primeira vez, o uso tradicional de plantas medicinais no município de Picos e demonstrou, também de forma inédita, a relevância de investimentos do Programa Farmácia Viva no município de Picos e sua inclusão no Programa Saúde da Família como forma de disponibilizar à população de baixa renda fitoterápicos produzidos localmente a custos reduzidos.
\end{abstract}

Palavras-chave: etnobotânica, uso popular, fitoterapia, toxicidade, Nordeste do Brasil.

\begin{abstract}
The therapeutic role of the Program Farmacia Viva and the medicinal plants in the center-south of Piauí. This study performed a research about the use of medicinal plants in Picos city, identifying the plants grown in the garden belonging to the Laboratory of Phytotherapics in Picos (LAFIPI) and analying the use of phytotherapics distributed by the Farmácia Viva Project between 2008-2010. From the total of 750 interviewed participants, $37.6 \%$ were men and $62.4 \%$ women. Most of them do not have secondary education $(69.2 \%)$ and $77.2 \%$ had an income of up until two minimum wages. Regarding consumption of medicinal plants, $76.3 \%$ used them to treat diseases, mainly because they considered them to be healthier $(84.8 \%)$. The indication of use of the plants was mainly suggested by relatives $(82.2 \%)$, even though the majority of the participants acquires plants in open markets (32.8\%). From the 127 plants mentioned, the most cited ones were balm, boldo and mint, and leaves were the most utilized parts $(42.3 \%)$, predominantly by infusion $(39.4 \%)$. The most common reasons for the usewere to treat pain in general $(17 \%)$, and respiratory (16.5\%) and digestive disorders $(16 \%)$. The most cultivated species in the garden were chambá (Justicia pectoralis), alecrim pimenta
\end{abstract}

Recebido para publicação em 03/04/2014 
(Lippia sidoides), malva santa (Plectranthus barbatus) and erva cidreira (Lippia alba). The "chambá licker" was the most herbal medicine searched by the population between 2008 and 2010. This investigation described, for the first time, the traditional use of medicinal plants in Picos and demonstrated,alsounprecedentedly, the relevance of investments in the Farmácia Viva Project in Picos city and its inclusion in the Family Health Program as a strategyin order to provide locally producedphytotherapics for low-income population at low costs.

Keywords: ethonobotany, folk use, phytotherapy, toxicity, Brazilian Northeast.

\section{INTRODUÇÃO}

Cerca de $80 \%$ da população mundial depende da medicina tradicional para suas necessidades básicas de saúde e quase $85 \%$ da medicina tradicional envolve o uso de plantas medicinais, seus extratos vegetais e seus princípios ativos (WHO, 2011). Entre 1981 e 2010, das 1.073 novas entidades químicas (New Chemical Entities - NCEs) aprovadas como medicamento pelo Food and Drug Administration (FDA) dos Estados Unidos, apenas $36 \%$ foram classificadas como verdadeiramente sintéticas, sendo que $64 \%$ são moléculas naturais, derivadas ou sintetizadas com base em compostos naturais. Apesar do interesse na modelagem molecular, na química combinatória e outras técnicas de síntese química, os produtos naturais e, particularmente, as plantas, permanecem, portanto, como uma importante fonte de novos agentes terapêuticos contra infecções (fúngicas ou bacterianas), radicais livres, mosquitos vetores de doenças, câncer, dislipidemias e imunomodulação (Butler, 2004; Balunas \& Kingnorn, 2005; Ferreira et al., 2009, 2011; Newman \& Cragg, 2012; Carvalho et al., 2013; Farias et al., 2013; Santos et al., 2013).

Segundo a Organização Mundial da Saúde (OMS), planta medicinal é todo vegetal que contém, em um ou vários de seus órgãos, substâncias que podem ser empregadas para fins terapêuticos ou precursores de substâncias utilizadas para tais propósitos (OMS, 2002). O fitoterápico, por sua vez, é o medicamento obtido empregando-se exclusivamente matérias-primas ativas vegetais e é caracterizado pelo conhecimento de sua eficácia e dos riscos do seu uso assim como pela reprodutibilidade e constância de sua qualidade (Brasil, 2004).

No Brasil, a finalidade curativa das plantas pode ser atribuída à diversidade de espécies vegetais e biomas, à riqueza étnico-cultural, ao modismo mundial recente de consumo de produtos considerados in natura e ao aumento de preço dos medicamentos industrializados (Garlet \& Irgang, 2001; Holetz et al., 2002; Veiga-Júnior et al., 2005; Ethur et al., 2011). O reconhecimento da importância de plantas para o desenvolvimento de fármacos modernos e terapeuticamente mais eficazes foi finalmente e nacionalmente oficializado pelo lançamento da Política Nacional de Plantas Medicinais e Fitoterápicos, aprovada por meio do Decreto № 5.813 em 22 de junho de 2006, a qual estabelece diretrizes para o desenvolvimento de ações voltadas à garantia do acesso seguro e uso racional de plantas medicinais e fitoterápicos, ao desenvolvimento de tecnologias e inovações, ao fortalecimento das cadeias e dos arranjos produtivos e ao uso sustentável da Biodiversidade Brasileira (Brasil, 2006).

Como uma forma de contribuir para a valorização da medicina popular no Estado do Piauí, o objetivo deste trabalho foi realizar um levantamento e registro sobre quais plantas são utilizadas como medicinais pela população urbana da cidade de Picos, levantar informações sobre a finalidade do uso, realizar a identificação taxonômica das plantas cultivadas no horto pertencente ao Laboratório Fitoterápico de Picos (LAFIPI) e descrever o uso de fitoterápicos dispensados pelo Programa Farmácia Viva do LAFIPI nos anos de 2008, 2009 e 2010.

\section{MATERIAL E MÉTODO Aplicação do questionário}

A primeira parte do estudo consistiu em um estudo de de caráter retrospectivo e transversal. Os estudos transversais apresentam-se como uma fotografia ou corte instantâneo que se faz numa população por meio de uma amostragem, examinando-se nos integrantes da casuística ou amostra, a presença ou ausência da exposição e a presença ou ausência do efeito (como a ação farmacológica ou tóxica, por exemplo). Já o estudo retrospective é realizado usando registros do passado, e é seguido adiante a partir daquele momento até o presente. É fundamental que haja credibilidade nos dados de registros a serem computados, em relação à exposição do fator e/ ou à sua intensidade, assim como pela ocorrência da doença ou situação clínica ou do óbito por esse motivo (Fletcher et al., 2003; Haddad, 2004). Assim, um questionário foi aplicado a 750 moradores residentes em sete diferentes bairros do município de Picos, Piauí (Bomba, Canto da Várzea, Centro, Junco, Pantanal, Parque de Exposição e Pedrinhas), 
e entre os meses de julho e setembro de 2011. O questionário foi previamente aplicado em um bairro da cidade para confirmar a adequação do instrumento e melhorar a qualidade das informações obtidas. Informações socioeconômicas, nome popular das plantas utilizadas, forma de utilização e finalidades do uso foram questionadas através de 13 perguntas contidas no questionário aplicado aos sujeitos das pesquisa. As visitas foram feitas em casas alternadas e após duas tentativas de entrevista sem sucesso, a visita à residência foi excluída. A distribuição quantitativa de domicílios incluídos na pesquisa foi proporcional à população de acordo com os dados disponibilizados pela Prefeitura Municipal de Picos. Após o aceite de participação na pesquisa, o entrevistado foi convidado a assinar um Termo de Consentimento Livre e Esclarecido (TCLE) em duas vias, ficando esse com a posse de uma dessas vias. Nenhum dado pessoal (como nome, endereço, telefone ou documentos de identificação) dos entrevistados foi registrado. Todos os estudos seguiram o estabelecido pelo Conselho Nacional de Saúde (Resolução №. 466/2012), pela declaração de Helsinque (Sousa et al., 2012) e pela Declaração Universal sobre Bioética e Direitos Humanos (UNESCO, 2006). A pesquisa foi aprovada em toda a sua plenitude pelo Comitê de Ética em Pesquisa da Universidade Federal do Piauí (Certificado de apresentação para Apreciação Ética - CAAE, no 0067.0.045.000-11). Nessa parte do estudo não houve coleta de espécimes vegetais.

\section{Apreciação do uso de fitoterápicos dispensados}

A coleta dos dados referentes à quantidade e o tipo de fitoterápico dispensado pelo Programa Farmácia Viva do LAFIPI foi realizada sob a supervisão da farmacêutica responsável e mediante autorização dos órgãos competentes, de forma retrospectiva e quantitativa com base nos arquivos de dispensação dos fitoterápicos registrados durante os anos de 2008, 2009 e 2010.

\section{Inventário das plantas medicinais cultivadas no LAFIPI}

A realização do inventário das plantas medicinais cultivadas no horto do LAFIPI foi baseada na coleta botânica de espécimes vegetais em estágio reprodutivo. Estas coletas seguiram a metodologia usual em taxonomia de fanerógamas (Mori et al., 1989). Após a coleta e a herborização, o material foi depositado no Herbário de Picos e as duplicatas foram enviadas para o acervo do Herbário Graziela Barroso (TEPB) da Universidade Federal do Piauí (UFPI).

A identificação dos taxa foi baseada no uso de chaves de identificação para famílias Souza \&
Lorenzi (2005) assim como no uso de chaves de identificação específicas de acordo com as famílias das plantas inventariadas. A padronização das abreviações dos nomes dos autores das plantas seguiram Brummitt \& Powell (1992).

\section{Análise do Dados}

Os resultados foram expressos em valores percentuais e apresentados em figuras produzidas no programa Prisma versão 4.0 (GraphPad, Intuitive Software for Science, San Diego, CA) e Excel. Os valores percentuais foram usados para definir qualitativamente o perfil da população que faz uso de plantas medicinais levando em consideração o ambiente natural como fonte direta dos dados e o pesquisador como instrumento fundamental, o caráter descritivo, o significado que as pessoas dão às coisas e à sua vida e o enfoque indutivo (Godoy, 1995).

\section{RESULTADO E DISCUSSÃO}

A busca do aproveitamento racional dos recursos naturais e a conservação de suas milenares culturas e tradições contribuem para um aplicação adequada e sustentável do ambiente, para a preservação desses recursos e disponibilizam às gerações futuras a possibilidade de conhecer, valorizar e se beneficiar do patrimônio que é a biodiversidade (Kiill et al., 2007; Pasa \& Ávila, 2010). Porém, a desagregação dos sistemas de vida tradicionais que acompanham a devastação do ambiente e a inclusão de novos elementos culturais ameaçam o acervo dos conhecimentos empíricos e do patrimônio genético (Amorozo, 2002). Essa ameaça é evidente na cidade de Picos, graças à presença de pessoas de quase todos os estados brasileiros, atraídas pelo elevado crescimento econômico da região e grande fluxo de pessoas, sendo o município de Picos a segunda cidade do Piauí a recolher Impostos Sobre Circulação de Mercadorias e Serviços (ICMS) para o governo estadual e exercendo grande influencia socioeconômica na região centro-sul do Piauí e nos estados vizinhos (Ceará e Maranhão) (Fernandes \& Bezerra, 1990).

O levantamento do uso de plantas no tratamento de enfermidades permite conhecer suas propriedades curativas e as reações tóxicas associadas ao consumo inadequado e/ou exagerado e confirma que os conhecimentos e as culturas tradicionais podem contribuir para a conservação à longo prazo da biodiversidade dos ecossistemas (Amorozo, 2002; Silva et al., 2014). Esse estudo conservacional é essencial para regiões onde a preservação ambiental inexiste e a especulação imobiliária, muitas vezes, apoiada por governantes

Rev. Bras. PI. Med., Campinas, v.17, n.4, p.550-561, 2015. 
locais, direciona as formas de ocupação territorial, que por sua vez, determinam condições ambientais como moradia, adensamento populacional e saneamento ambiental.

Os dados obtidos a partir de $1 \%$ da população oficial coberta pela presente pesquisa mostraram que do total dos 750 entrevistados, $282(37,6 \%)$ foram representados por homens e $468(62,4 \%)$ por mulheres. A maior proporção de mulheres pode ser explicada pela ausência dos homens no domicílio no horário em que as entrevistas foram realizadas (quase sempre das 8 às 19h) já que a maioria das mulheres realiza somente trabalhos domésticos, não se distanciando de casa por um tempo muito prolongado ou devido ao fato de muitas residências serem chefiadas por mulheres. Resultados similares foram obtidos em Guamirim, uma comunidade rural do município paranaense de Irati, onde se observou que 83\% dos entrevistados eram do sexo feminino (Jacoby et al., 2002). A maioria das pessoas (composta por mulheres) que responderam ao questionário possuía entre 31 e 55 anos de idade (368 pessoas, $49 \%$ do total), enquanto $28,3 \%$ possuíam entre 18 e 30 anos, $18 \%$ entre 56 e 70 anos e apenas 4,4\% da população estudada possuía entre 71 e 85 anos.

Com relação ao nível de escolaridade, verificou-se que a maioria não concluiu o ensino fundamental $(44,1 \%)$, sendo que os analfabetos representaram $2,2 \%$. Por outro lado, apenas 3,8\% tinham graduação completa (Figura 1). Portanto, $69,2 \%(331+98+90=519)$ dos entrevistados nunca completaram o segundo grau. Sevignani \& Jacomassi (2003) também mostraram que a maioria dos seus entrevistados possuía ensino primário incompleto. A baixa escolaridade esteve associada a uma renda igualmente baixa, uma vez que $77,2 \%$ dos entrevistados possuíam uma renda mensal de até 2 salários mínimos, $21,1 \%$ dos entrevistados recebiam entre 2 e 5 salários e apenas $1,7 \%$ dos entrevistados recebia entre 5 e 10 salários mínimos. Observou-se, previamente, que o conhecimento sobre plantas medicinais tem uma tendência a diminuir com o nível de escolaridade (Santos et al, 2008) e como o nível de escolaridade tem relação com o poder aquisitivo, o baixo nível de escolaridade e conhecimento popular muitas vezes refletem no uso das plantas medicinais como alternativa para os medicamentos alopáticos de alto custo.

Com relação à utilização de plantas medicinais, $76,3 \%$, ou seja, 572 dos 750 entrevistados afirmaram utilizá-las para tratar ou curar doenças, principalmente por considerarem mais saudáveis ( $84,8 \%$ do total) quando comparadas aos medicamentos alopáticos, embora $7,5 \%$ dos entrevistados afirmam usar produtos vegetais por serem mais baratos. O amplo uso de plantas medicinais para tratar patologias foi previamente relatado por Veiga-Júnior (2008), mostrando que $97,7 \%$ dos entrevistados utilizam plantas para fins terapêuticos.

Quando questionados com quem aprenderam a utilizar tais plantas, $82,2 \%$ responderam ter sido com a família e $13,5 \%$ com amigos, mostrando que o uso de plantas é passado de geração a geração, sugerindo a existência de uma cultura local da população em semelhança aos resultados encontrados no município de Datas (MG), onde $84,5 \%$ dos entrevistados afirmaram ter aprendido sobre plantas medicinais com seus antecedentes (Arnous et al., 2005). Assim, a difusão das propriedades farmacológicas das plantas tem sido realizada principalmente pela língua falada, de forma simples, no dia a dia, sendo que, na maioria

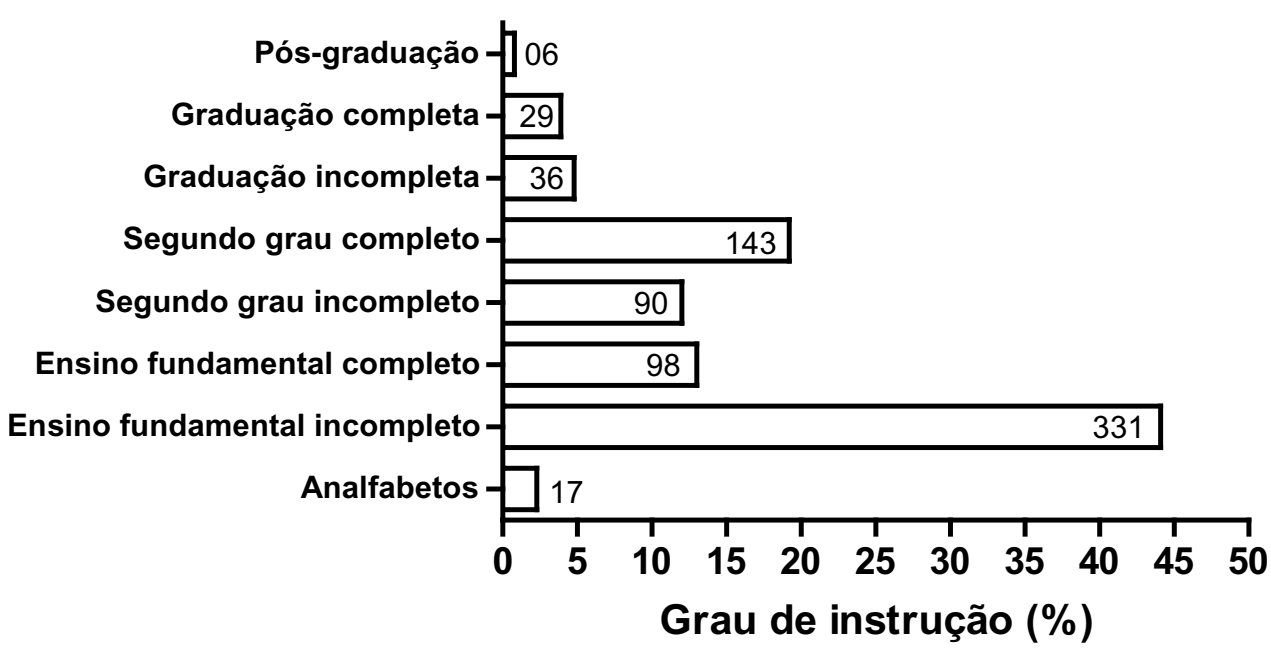

FIGURA 1. Grau de instrução dos entrevistados na cidade de Picos $(n=750)$. Os valores dentro das barras correspondem ao número de entrevistados. 
das vezes, o aprendizado sobre o tema é transferido de pai/mãe ou avô/avó para filho(a), compartilhando o saber tradicional teórico e prático adquiridos e, ao mesmo tempo, acolhendo as explicações, com seus mitos e crenças (Veiga-Júnior et al., 2005).

É importante relatar que os profissionais de saúde (biólogos, enfermeiros, farmacêuticos, dentistas, médicos e nutricionistas) na cidade de Picos possuem um pequeno papel na propagação ou aconselhamento correto sobre o consumo de fitoterápicos ou de preparações vegetais para o tratamento de doenças, uma vez que somente 1,3\% dos questionados relataram possuir tal atendimento ou indicação especializada. O desconhecimento das propriedades biológicas das plantas mais usadas e a carência de pesquisas sobre estudos de farmacocinética, farmacodinâmica e toxicologia são os principais fatores que desencorajam os profissionais de saúde a receitar e aceitar os fitoterápicos como um recurso terapêutico.

Observou-se, no presente trabalho, que a maioria dos entrevistados adquire as plantas em feiras livres $(32,8 \%)$ da cidade de Picos ou no quintal de suas casas $(25,6 \%)$ e nos supermercados $(18,3 \%)$ (Figura 2$)$. Somavilla \& Canto-Dorow (1996) verificaram que $76 \%$ das plantas utilizadas como medicinais na cidade de Santa Maria (RS) são obtidas através de amigos e também pelo hábito de cultivo caseiro. Resultados similares também foram encontrados por Viganó \& Silva (2007) no qual a maioria dos entrevistados cultiva as plantas no quintal de casa (44\%) ou são obtidas por intermédio de familiares ou amigos. De fato, os quintais, considerados sistemas agroflorestais importantes para o cultivo, seleção e conservação de espécies de plantas, fornecem recursos para o consumo familiar e preservam a cultura local
(Kumar \& Nair, 2004), sendo um espaço de grande relevância para o contato com distintas espécies vegetais e manutenção dos conhecimentos populares Além disso, os quintais facilitam as ações antropogênicas, principalmente no que se refere às condições de sobrevivência nos períodos de seca do sertão nordestino, onde algumas plantas perdem suas folhas e outras chegam a morrer (Siviero et al., 2011). Para Amorozo (2002), o hábito de cultivar plantas em quintais nas zonas urbanas permite formar ilhas de vegetação por meio da união de vários espaços plantados, como praças e parques, que contribuem para melhorar a qualidade de vida nos centros urbanos. Por outro lado, nas regiões mais pobres do país e até mesmo nas grandes cidades brasileiras, plantas medicinais são comercializadas em feiras livres e mercados populares por raizeiros e ervanários. Essas plantas costumam apresentar uma queda da qualidade quanto à análise farmacológica devido a erros de classificação botânica, época e forma de coleta, secagem, acondicionamento e contaminação por fungos e outros microrganismos (Amorozo, 2002; Nunes et al., 2003; Ustulin et al., 2009).

Das 127 plantas medicinais citadas pela população pesquisada por meio da aplicação do questionário, as que tiveram maior destaque foram: erva-cidreira (292 citações), boldo (225), hortelã (224), eucalipto (149), capim santo (149), erva doce (134), malva do reino (132) e malva santa (100). Os resultados são condizentes com os encontrados em estudo realizado em São Paulo (Pinto et al., 2006), o qual cita como plantas mais utilizadas o boldo, capim-santo e hortelã e com os estudos descritos por Jacoby et al. (2002) e Costa \& Mayworm (2011), onde são citadas também a erva-cidreira, boldo e hortelã-pimenta.

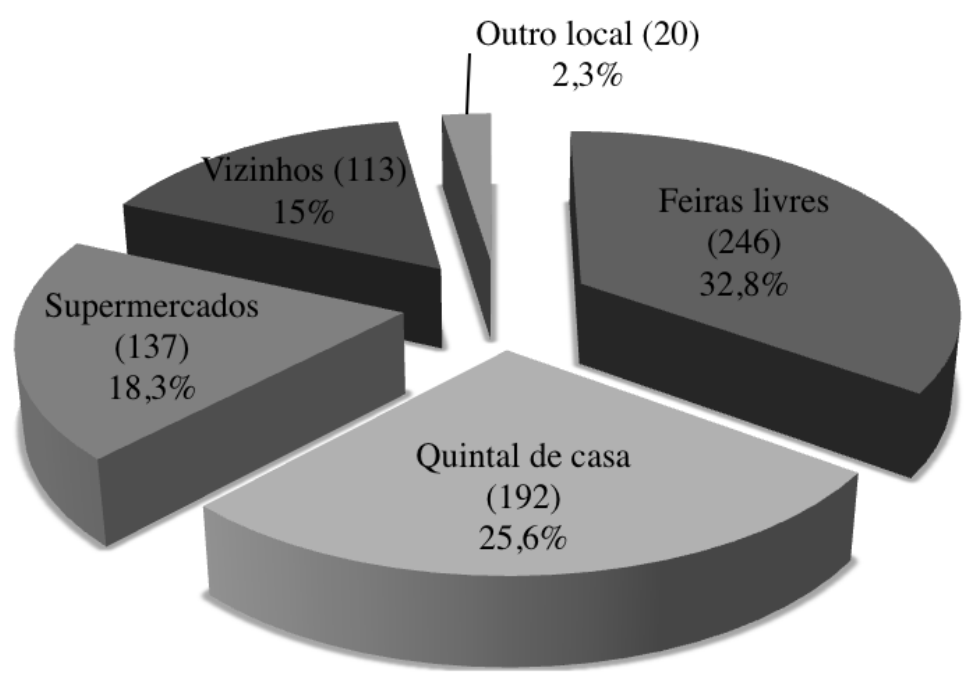

FIGURA 2. Locais de obtenção das plantas medicinais ou partes delas para consumo pessoal pela população de Picos. Cada pessoa teve a liberdade de citar mais de um local de obtenção. 
No que se refere à parte utilizada, encontrou-se grande uso das folhas $(42,3 \%)$, além das sementes $(14,6 \%)$ e casca $(12,9 \%)$ (Figura 3 ). Jacoby et al. (2002), Santos et al. (2008) e Costa \& Mayworm (2011) também constataram que a folha foi parte da planta mais utilizada. A utilização mais freqüente das folhas está associada a uma coleta menos laboriosa e maior disponibilidade ao longo do ano (Castellucci et al., 2000). Outra razão seria o fato de que os mecanismos químicos e, portanto, os princípios ativos, concentram-se nas partes da planta que mais contribuem para o desenvolvimento como folhas novas, ramos de florescência e sementes (Cheeke, 1998).

Em relação à forma de utilização, observouse a predominância da infusão $(39,4 \%$ ou 452 citações), além do consumo de lambedores (19,9\%) e de preparações por decocção (16,8\%) (Figura 4). Conforme já anteriormente observado por Veiga-Júnior(2008), 60,2\% dos seus entrevistados utilizam plantas por meio de chás e infusões. Também é oportuno destacar que a decocção deve ser realizada com as partes mais rígidas da planta como caule e raízes, ao passo que a infusão com as mais sensíveis como folhas e flores, sendo o chá um tipo de decocção não muito indicado para estas últimas, pois a fervura contínua pode causar a perda de alguns princípios ativos (Sevignani \& Jacomassi, 2003). Logo, saber distinguir morfologicamente e até quimicamente a parte do vegetal a ser empregada é extremamente importante, pois os princípios ativos distribuem-se pelas partes da planta de forma distinta, sendo possível encontrar também substâncias tóxicas em algumas dessas partes (Pinto et al., 2006).

Quanto às reações indesejadas, apenas $1,2 \%$ dos entrevistados relataram mal-estar após consumo de produtos vegetais para o tratamento de enfermidades, reforçando ainda mais o conceito popular equivocado de que plantas medicinais não fazem mal à saúde, o que pode ser considerado um fator de risco para usuários, uma vez que as plantas possuem diversos compostos (alcalóides, glicosídeos, lecitinas e ácidos orgânicos) com comprovado potencial tóxico (Cheeke, 1998). Observa-se que a maioria dos efeitos colaterais registrados está relacionada com a identificação incorreta das plantas, necessidade de padronização, prática deficiente de processamento, contaminação, substituição e adulteração de plantas, preparação e/ou dosagem incorretas (Calixto, 2000).

Constatou-se ainda que as principais finalidades da utilização das plantas medicinais pela população são: dores em geral (17\%), problemas do sistema respiratório $(16,5 \%)$ e problemas digestivos (16\%) (Figura 5). Em Itacaré, na Bahia, as doenças mais tratadas com produtos naturais originados de plantas são também aquelas referentes ao aparelho digestivo $(21,4 \%)$, sintomas e sinais gerais $(15,4 \%)$ e problemas do aparelho respiratório (10,8\%) (Somavilla \& Canto-Dorow, 1996). Justamente as plantas mais citadas estão diretamente interligadas às finalidades mais lembradas pelos entrevistados como a erva-cidreira, usada como calmante e contra cólicas, febre, dores de cabeça, tosse e para reduzir a pressão arterial; a hortelã, usada

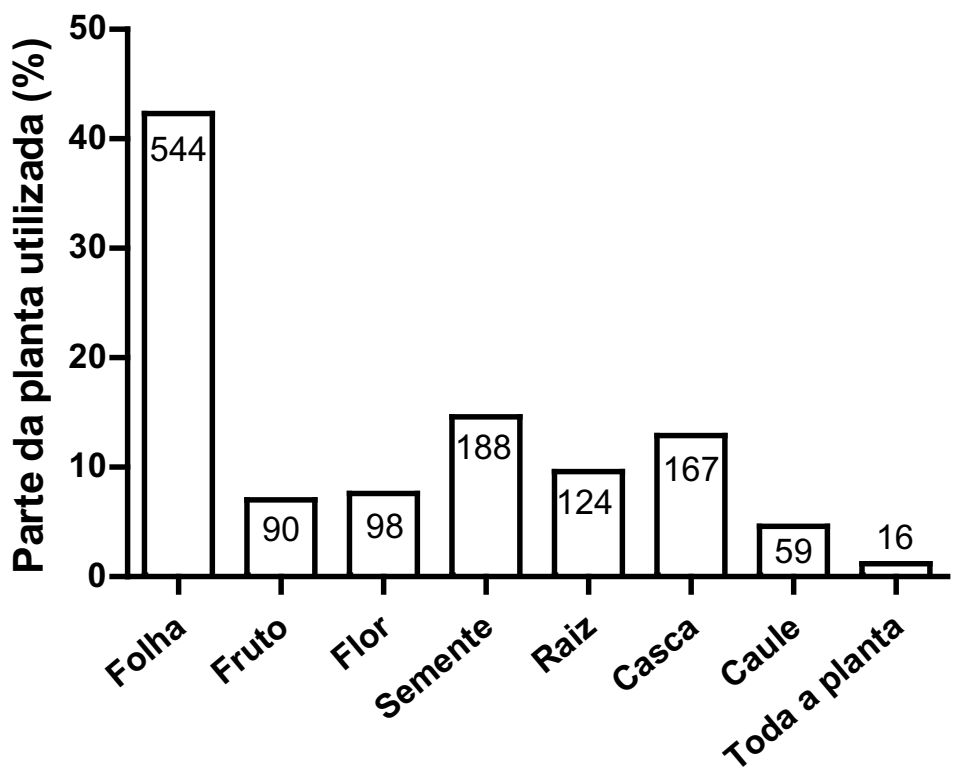

FIGURA 3. Partes das plantas mais utilizadas para o consumo com fins terapêuticos pela população de Picos. Cada pessoa teve a liberdade de citar a(s) parte(s) da planta que tenha o costume de consumir. 


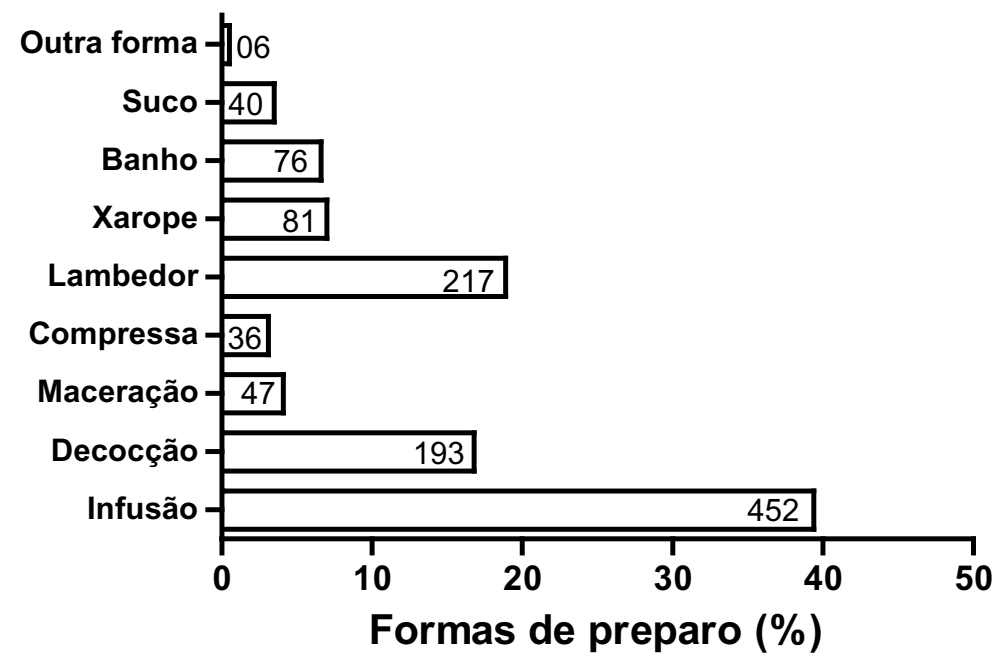

FIGURA 4. Formas de preparo das plantas para consumo pela população de Picos. Cada pessoa teve a liberdade de citar as mais diversas formas de preparo que tenha o costume de utilizar para consumir a planta ou partes dela.

como vermífugo, digestivo e em gripes; a erva doce, usada como antiespasmódica, expectorante, calmante e contra prisão de ventre; e o boldo, para tratar distúrbios gastrointestinais e usado também como um tranquilizante para o sono(Almeida, 1993; Sevignani \& Jacomassi, 2003).

Outro achado interessante é que $69,5 \%$ dos entrevistados (404 das pessoas) afirmaram que não há diferença da utilização das plantas medicinais entre os períodos seco e chuvoso, embora $16,7 \%$ afirmaram utilizar mais em período chuvoso e $14,2 \%$ utilizam mais em período seco (entre os meses de abril a novembro).

O uso disseminado de produtos vegetais com propriedades terapêuticas pela população se confirmou com os estudos de dispensação de fitoterápicos pelo LAFIPI, observando-se uma grande procura durante todos os anos avaliados (1776, 1647 e 1396 dispensações nos anos de 2008, 2009 e 2010, respectivamente). Acredita-se

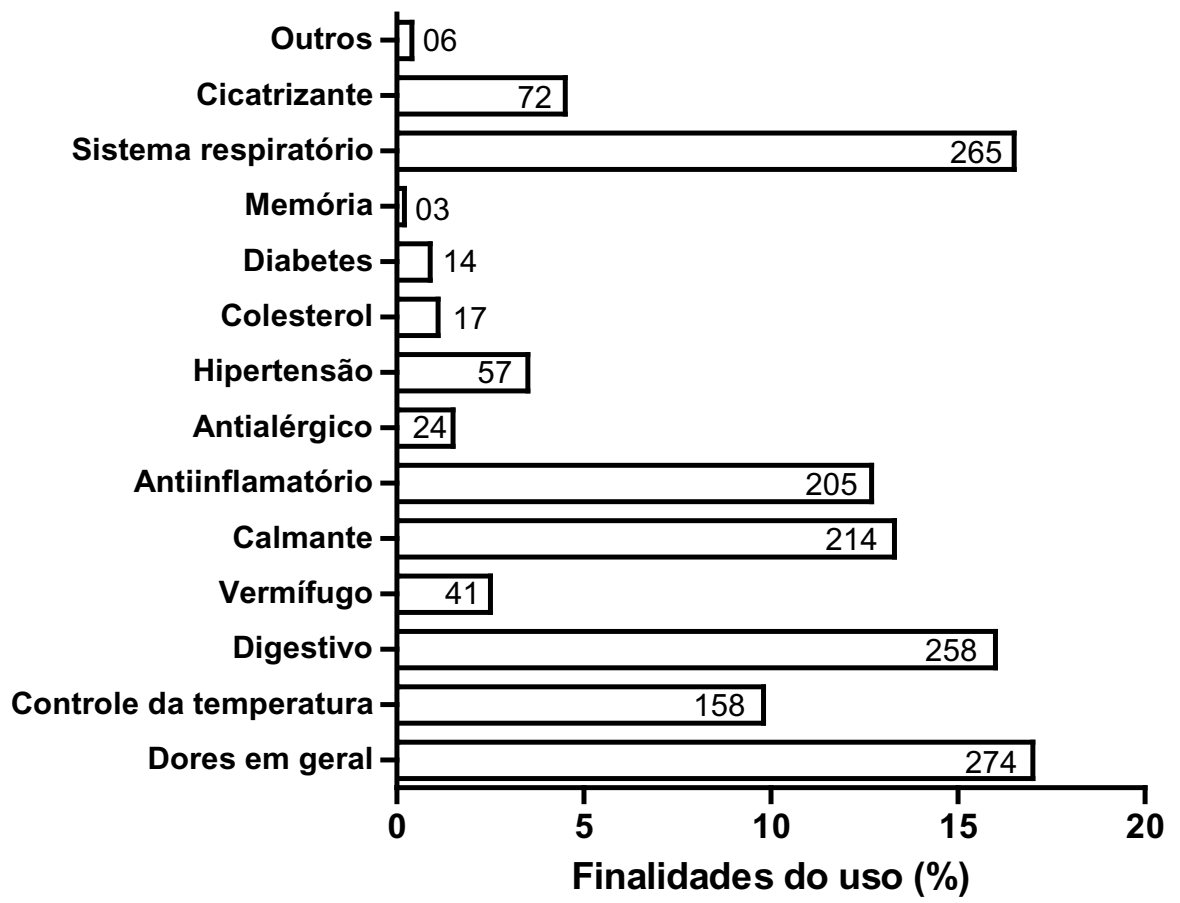

FIGURA 5. Principais finalidades da utilização de plantas medicinais pela população de Picos. Cada pessoa teve a liberdade de citar mais de uma finalidade terapêutica. 
que essa queda na dispensação durante os anos avaliados deva-se à mudança de endereço do LAFIPI e dificuldades financeiras para a produção dos fitoterápicos, uma vez que não há subsídios fixos de órgãos governamentais ou privados para tal produção, ficando, assim, o Programa Farmácia Viva dependente de contribuições esporádicas.

A experiência mais antiga que influenciou a criação de programas de fitoterapia no Brasil foi o Programa Farmácia Viva criado pelo professor Francisco José de Abreu Matos da Universidade Federal do Ceará, há mais de vinte anos. O município de Maracanaú (CE) foi o primeiro a implantar o Programa Farmácia Viva no Sistema Público de Saúde. Atualmente, o programa está vinculado à Secretaria Municipal de Saúde e tem se destacado pela organização e abrangência, A dispensação dos produtos à comunidade ocorre nas Unidades Básicas de Atenção à Saúde da Família do Programa Saúde da Família (PSF), mediante prescrição medicamentosa do profissional de saúde. O Programa Farmácia Viva de Picos tem ganhado reconhecimento pela população embora poucas sejam as aquisições governamentais para o Programa. Além disso, o Programa Farmácia Viva de Picos ainda não foi inserido no PSF, o que dificulta a atração de investimentos para ampliar e otimizar sua funcionalidade. Os fitoterápicos produzidos e dispensados pelo LAFIPI são oriundos de plantas cultivadas em canteiros no horto do LAFIPI, as quais foram coletadas, inventariadas, identificadas, herborizadas e registradas na coleção do Herbário Graziela Barroso (TEPB) da Universidade Federal do Piauí,
Campus Ministro Petrônio Portela, Teresina, Piauí, Brasil (Figura 6). O horto apresentou-se como uma área de cultivo organizada em canteiros onde se encontravam dez espécies pertencentes a seis famílias botânicas, destacando-se a família Lamiaceae com quatro espécies (Tabela 1).

As espécies mais cultivadas no horto são chambá (Justicia pectoralis Jacq.), alecrim pimenta (Lippia sidoides Cham.), malva santa (Plectranthus barbatus Andrews) e erva cidreira (Lippia alba (Mill.) N.E. Br. ex Britton \& P. Wilson), as quais são utilizadas como principais fontes dos princípios ativos dos fitoterápicos dispensados pelo LAFIPI (lambedor de chambá, tintura de alecrim-pimenta, tintura de malva santa e cápsula de cidreira, respectivamente) (Figura 7). Além dessas plantas, pode-se observar que plantas como hortelã (Mentha $\times$ villosa Huds), pata de vaca (Bauhinia ungulata L.), pimenta dos monges (Vitex agnus-castus L.), romã (Punica granatum L.), alfavaca cravo (Ocimum gratissimum L.) e babosa [Aloe vera (L.) Burm. f.] também são cultivadas no horto e são fornecidas à população local de forma gratuita. Assim, como visto na Figura 7 , o lambedor de chambá foi o fitoterápico mais procurado pela população entre 2008 e 2010. Observou-se também que houve uma maior frequência de uso destes fitoterápicos entre os meses de abril e novembro, um fato provavelmente relacionado às altas temperaturas e repentinas mudanças climáticas e coincidindo com a época mais quente no semiárido brasileiro (agosto a novembro). Seguramente, o uso do lambedor de chambá envolve as indicações terapêuticas de $J$. pectoralis, principalmente, aquelas destinadas ao

TABELA 1. Lista de plantas medicinais cultivadas no horto do Laboratório Fitoterápico de Picos (LAFIPI), devidamente catalogadas e identificadas com seus respectivos nomes populares, famílias botânicas e o número de registro no Herbário Graziela Barroso (TEPB).

\begin{tabular}{lccc}
\hline Nome Popular & Família & Espécie & Voucher \\
\hline Pata de Vaca & Fabaceae & Bauhinia ungulata L. & TEPB 28.281 \\
Hortelã & Lamiaceae & Mentha x villosa Huds. & TEPB 28.275 \\
Chambá & Acanthaceae & Justicia pectoralis Jacq. & TEPB 28.274 \\
Erva cidreira & Verbenaceae & Lippia alba (Mill.) N.E. Br. ex Britton \& P. Wilson & TEPB 28.276 \\
Malva santa & Lamiaceae & Plectranthus barbatus Andrews & TEPB 28.280 \\
Alecrim pimenta & Verbenaceae & Lippia sidoides Cham. & TEPB 28.278 \\
Alfavaca cravo & Lamiaceae & Ocimum gratissimum L. & TEPB 28.277 \\
Pimenta dos & Lamiaceae & Vitex agnus-castus L. & TEPB 28.272 \\
monges & & & \\
Romã & Lythraceae & Punica granatum L. & TEPB 28.270 \\
Babosa & Xanthorrhoeaceae & Aloe vera (L.) Burm. f. & TEPB 28.269 \\
\hline
\end{tabular}

Rev. Bras. PI. Med., Campinas, v.17, n.4, p.550-561, 2015. 

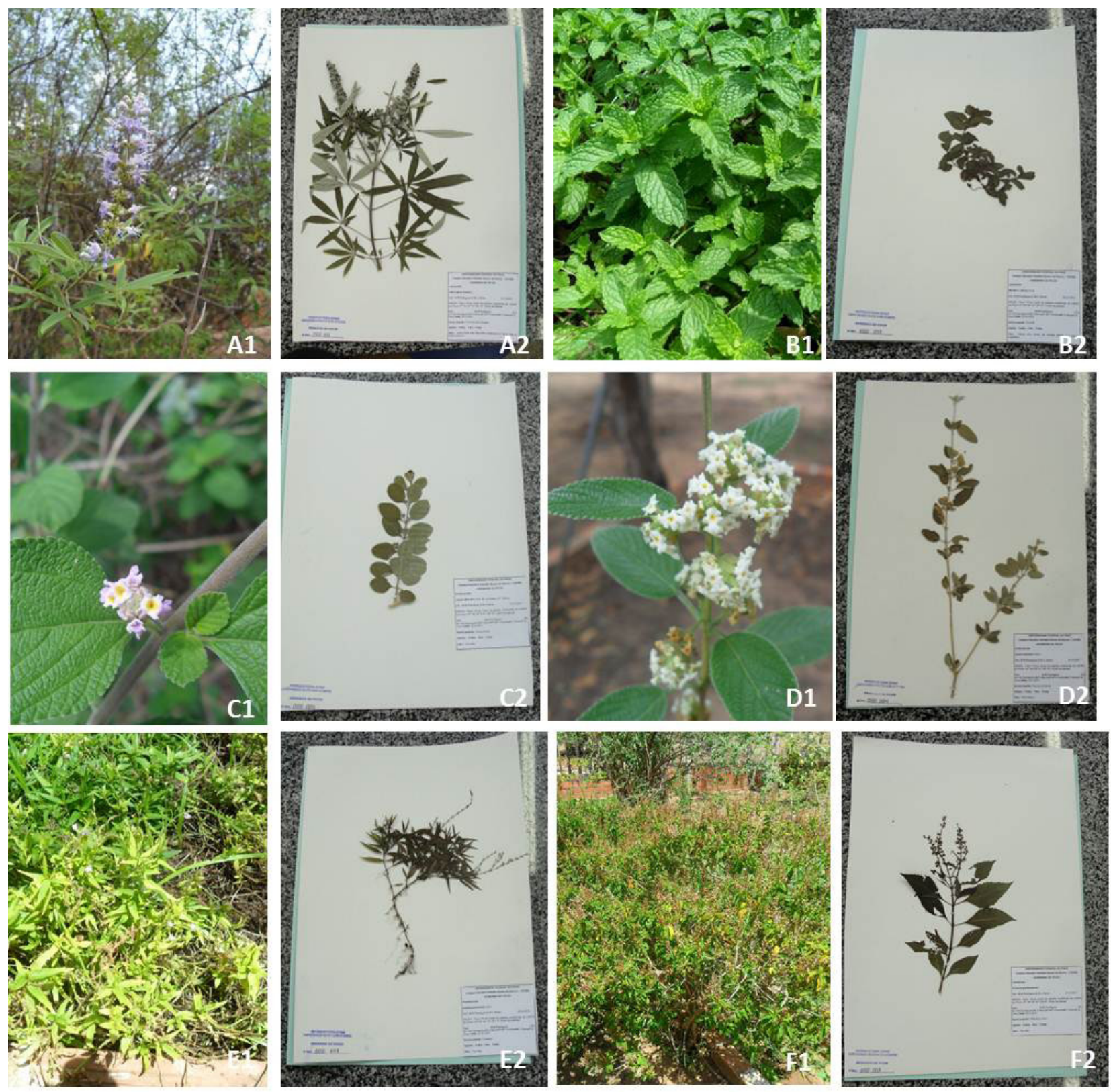

FIGURA 6. Espécies cultivadas no horto do Laboratório Fitoterápico de Picos (LAFIPI) do Programa Farmácia Viva e suas respectivas exsicatas. A1e A2: Pimenta dos monges (Vitex agnus-castus L.), B1 e B2: Hortelã (Mentha $\times$ villosa Huds.), C1 e C2: Erva cidreira (Lippia alba (Mill.) N.E. Br. ex Britton \& P. Wilson), D1 e D2: Alecrim pimenta (Lippia sidoides Cham.), E1 e E2: Chambá (Justicia pectoralis Jacq.), F1 e F2: Alfavaca cravo (Ocimum gratissimum L.).

tratamento de patologias do sistema respiratório devido às propriedades broncodilatadora e antiinflamatória (Oliveira et al., 2000; Morais et al., 2005).

Os resultados detalhados no presente trabalho mostram ineditismo em muitos aspectos, uma vez que poucos estudos têm sido realizados acerca do uso de plantas medicinais pela população piauiense. Medeiros (2001) e Medeiros et al. (2007) publicaram trabalhos de caráter descritivo e qualitativo de maneira a comparar saberes científicos e populares no uso de plantas medicinais em condições de saúde-doença no estado do Piauí.

É importante destacar que o conhecimento sobre plantas pode sofrer alterações à medida que a relação com a terra se transforma pela modernização do campo e os contatos com culturas diferentes (nacionais ou estrangeiras) se intensificam seja pelos meios de comunicação ou por agentes sociais, enfatizando que as pesquisas sobre o uso popular das plantas deve ser um contínuo, principalmente quando se fala sobre a extração e prospecção realizadas diretamente de florestas, parques e bosques (legalmente protegidos ou não) (Pilla et al, 


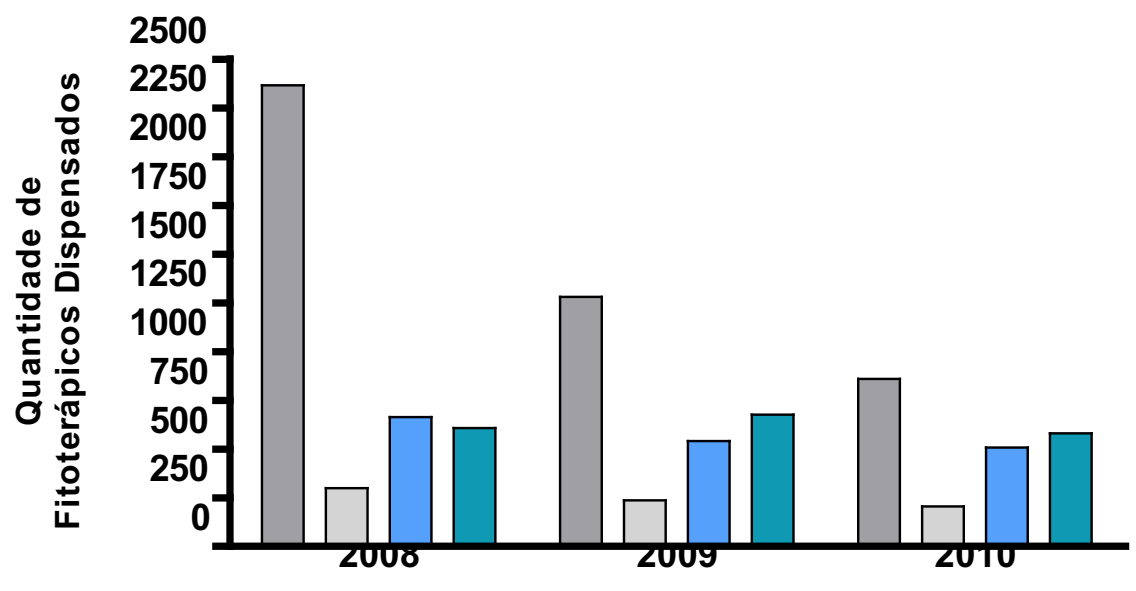

$\square$ Lambedor de Chambá Tintura de Malva Santa Tintura de Alecrim Pimenta Cápsula de Cidreira

FIGURA 7. Quantidade de fitoterápicos dispensados pelo Laboratório de Fitoterápicos de Picos (LAFIPI) nos anos de 2008, 2009 e 2010.

2006). Paula (2000) reforça que a coleta correta de cada órgão da planta deve ser orientada de forma a causar o menor impacto possível sob o ponto de vista de sustentabilidade, manejo e utilização.

Os resultados mostram que o perfil da população estudada que faz uso de plantas medicinais é representada por uma maioria de adultos, mulheres de baixo poder aquisitivo, idade entre 31-55 anos e ensino fundamental incompleto. A principal forma de obtenção das plantas ocorre nas feiras livres do próprio município, com o propósito de tratar principalmente distúrbios do sistema respiratório e digestivo, utilizando as folhas preparadas predominantemente por infusão. O lambedor de chambá foi o fitoterápico mais procurado pela população no LAFIPI no triênio 20082010. Esse estudo permitiu ainda a comprovação do uso de plantas medicinais no centro-sul piauiense e constatou a correlação entre o saber tradicional e o científico, cada vez mais respaldado e evidenciado em várias regiões no Brasil. Além disso, demonstrou, pela primeira vez, a relevância de investimentos no Programa Farmácia Viva de Picos e a importância de sua inclusão no PSF como uma forma de disponibilizar fitoterápicos de origem local à população de baixa renda a custos reduzidos.

\section{AGRADECIMENTO}

À Fundação de Amparo à Pesquisa do Estado do Piauí (FAPEPI) e ao Conselho Nacional de Desenvolvimento Científico e Tecnológico (CNPq) pelo apoio na forma de bolsas de estudo.

\section{REFERÊNCIAS}

ALMEIDA, E.R. Plantas medicinais brasileiras: conhecimentos populares e científicos. São Paulo: Hemus Editora Ltda, 1993. 341p.

AMOROZO, M.C.M. Uso e diversidade de plantas medicinais em Santo Antônio do Leverger, MT, Brasil. Acta Botanica Brasilica, v.16, n.2, p.189-203, 2002.

ARNOUS, A.H. et al. Plantas medicinais de uso caseiro - conhecimento popular e interesse por cultivo comunitário. Revista Espaço para a Saúde, v.6, n.2, p.1-6, 2005.

BALUNAS, M.J.; KINGNORN, A.D. Drug discovery from medicinal plants. Life Sciences, v.78, n.5, p.431-41, 2005.

BRASIL. Ministério da Saúde. Agência Nacional de Vigilância Sanitária. Resolução de Diretoria Colegiada no. 48 de 16 de março de 2004. Aprova o regulamento técnico de medicamentos fitoterápico junto ao Sistema Nacional de Vigilância Sanitária. DOU. Diário Oficial da União, Poder Executivo, DF, Brasília, 2004.

BRASIL. Ministério da Saúde. Secretaria de Ciência, Tecnologia e Insumos Estratégicos. Departamento de Assistência Farmacêutica. Política Nacional de Plantas Medicinais e Fitoterápicos. Brasília: Série B - Textos Básicos de Saúde, 2006. 60p.

BRUMMITT, R.F.; POWELL, C.E. Authors of plant names. Londres: Royal Botanic Gardens-Kew, 1992. $732 p$.

BUTLER, M.S. The role of natural product chemistry in drug discovery. Journal of Natural Products, v.67, n.12, p.2141-153, 2004.

CALIXTO, J.B. Efficacy, safety, quality control, marketing and regulatory guidelines for herbal medicines (phytotherapeutic agents). Brazilian Journal of Medical and Biology Research, v.33, n.2, p.179-89, 2000. 
CARVALHO, C.C. et al. Biological screening of extracts of Brazilian Asteraceae plants. African Journal of Pharmacy and Pharmacology, v.7, n.28, p.2000-5, 2013.

CASTELLUCCI, S. et al. Plantas medicinais relatadas pela comunidade residente na Estação Ecológica de Jataí, município de Luís Antônio - SP: uma abordagem etnobotânica. Revista Brasileira de Plantas Medicinais, v.3, n.1, p.51-60, 2000.

CHEEKE, P.R. Natural toxicants in feeds, forages, and poisonous plants. 2ed. Danville: Interstate Publishers, 1998. 479p.

COSTA, V.P.; MAYWORM, M.A.S. Plantas medicinais utilizadas pela comunidade do bairro dos Tenentes município de Extrema, MG, Brasil. Revista Brasileira de Plantas Medicinais, v.13, n.3, p.282-92, 2011.

ETHUR, L.Z. et al. Comércio formal e perfil de consumidores de plantas medicinais e fitoterápicos no município de Itaqui - RS. Revista Brasileira de Plantas Medicinais, v.13, n.2, p.121-28, 2011.

FARIAS, D.F. et al. Antibacterial, antioxidant, and anticholinesterase activities of plant seed extracts from Brazilian semiarid region. BioMed Research International, v. 2013, n. 510736, p.1-9, 2013.

FERNANDES, A.; BEZERRA, P. Estudo Fitogeográfico do Brasil. Fortaleza: Stylus Comunicações, 1990. 205p.

FERREIRA, P.M.P. et al. Larvicidal activity of the water extract of Moringa oleifera seeds against Aedes aegypti and its toxicity upon laboratory animals. Anais da Academia Brasileira de Ciências, v.81, n.2, p.20716, 2009.

FERREIRA, P.M.P. et al. Folk uses and pharmacological properties of Casearia sylvestris: a medicinal review. Anais da Academia Brasileira de Ciências, v.83, n.4, p.1373-84, 2011.

FLETCHER, R.H.; FLETCHER, S.W.; WAGNER, E.H. Epidemiologia clínica: elementos essenciais. Porto Alegre: Artmed, 2003. 264p.

GARLET, T.M.B.; IRGANG, B.E. Plantas medicinais utilizadas na medicina popular por mulheres trabalhadoras rurais de Cruz Alta, Rio Grande do Sul, Brasil. Revista Brasileira de Plantas Medicinais, v.4, n.1, p.9-18, 2001.

GODOY, A.S. Introdução à Pesquisa qualitativa e suas possibilidades. Revista de Administração de Empresas, v.35, n.2, p.57-63, 1995.

HADDAD, N. Metodologia de estudos em ciências da saúde. Roca: Sao Paulo, 2004. 287p.

HOLETZ, F.B. et al. Screening of some plants used in the Brazilian folk medicine for the treatment of infectious diseases. Memórias do Instituto Oswald Cruz, v.97, n.7, p.1027-31, 2002.

JACOBY, C. et al. Plantas medicinais utilizadas pela comunidade rural de Guamirim, Município de Irati, PR. Revista de Ciências Exatas e Naturais, v.4, n.1, p.79-89, 2002.

KIILL, L.H.P. et al. Preservação e uso da Caatinga. Brasília: Embrapa Informação Tecnológica, 2007. 36p.

KUMAR, B.M.; NAIR, P.K.R. The enigma of tropical home gardens. Agroforestry Systems, v.61, n.1, p.135-52, 2004.

MEDEIROS, L.C.M. As plantas medicinais e a enfermagem - $\mathbf{a}$ arte de assistir, de curar, de cuidar e de transformar os saberes. Rio de Janeiro, 2001. 165p. Tese (Doutorado em Enfermagem) - Escola de Enfermagem Anna Néri, Universidade Federal do Rio de Janeiro, Rio de Janeiro.

MEDEIROS, L.C.M. et al. As práticas populares de cura utilizadas por rezadores no povoado Brejinho, município de Luiz Correia - PI. Escola Anna Nery Revista de Enfermagem, v.11, n.1, p.112-7, 2007.

MORAIS, S.M.; et al. Plantas medicinais usadas pelos índios Tapebas do Ceará. Revista Brasileira de Farmacognosia, v.15. n.2, p.169-77, 2005.

MORI, S.A.; SILVA, L.A.M.; LISBOA, G.; CORANDIN, L. Manual de manejo do herbário fanerogâmico. Ilhéus: Centro de Pesquisas do Cacau, 1989. 103p.

NEWMAN, D.J.; CRAGG, G.M., Natural products as sources of new drugs over the 30 years from 1981 to 2010. Journal of Natural Products, v.75, n.3, p.31135, 2012.

NUNES, G.P. et al. Plantas medicinais comercializadas por raizeiros no Centro de Campo Grande, Mato Grosso do Sul. Revista Brasileira de Farmacognosia, v.13, n.2, p.83-92, 2003.

OLIVEIRA, A.F.H; XAVIER, A.S.; SILVA, N.H.; ANDRADE, L.H.C. Screening cromatográfico de Acanthaceae medicinais: Justicia pectoralis Jacq. e J. Gendarussa Burm. Bras. PI. Med, v.3, n.1, p.37-41, 2000.

ORGANIZACIÓN MUNDIAL DE LA SALUD (OMS). Estrategia de la OMS sobre medicina tradicional 2002-2005. Genebra: OMS, 2002. 74p.

ORGANIZAÇÃO DAS NAÇÕES UNIDAS PARA A EDUCAÇÃO, CIÊNCIA E CULTURA (UNESCO). Declaração Universal sobre Bioética e Direitos Humanos. Lisboa: UNESCO. 2006. 12p.

PASA, M.C.; ÁVILA, G. Ribeirinhos e recursos vegetais: a etnobotânica em Rondonópolis, Mato Grosso, Brasil. Interações, v.11, n.2, p.195-204, 2010.

PAULA, J.E. Manejo Sustentável. Cadernos do Centro de Estudos Avançados Multidisciplinares da UnB, v.1, n.3, p.115-6, 2000.

PILLA, M.A.C. et al. Obtenção e uso de plantas medicinais no distrito de Martim Francisco, município de MogiMirim, SP, Brasil. Acta Botanica Brasilica, v.20, n.4, p.789-802, 2006.

PINTO, E.P.P. et al. Conhecimento popular sobre plantas medicinais em comunidades rurais de mata atlântica Itacaré, BA, Brasil. Acta Botanica Brasilica, v.20, n.4, p.751-62, 2006.

SANTOS, M.R.A. et al. Uso de plantas medicinais pela população de Ariquemes, em Rondônia. Horticultura Brasileira, v.26, n.2, p.244-50, 2008.

SANTOS, A.C.B. et al.. Levantamento etnobotânico, químico e farmacológico de espécies de Apocynaceae Juss. ocorrentes no Brasil. Revista Brasileira de Plantas Medicinais, v.15, n.3, p.442-58, 2013.

SOUSA, M.S.A. et al. The new declaration of Helsinki and the use of placebo in brazilian clinical trials: controversy remains. Revista de Medicina, v.91, n.3, p.178-88, 2012.

SEVIGNANI, A.; JACOMASSI, E. Levantamento de plantas medicinais e suas aplicações na Vila Rural "Serra dos Dourados" - Umuarama - PR. Arquivos de Ciências da Saúde da Unipar, v.7, n.1, p.27-31, 2003. SILVA, L.R.R. et al. Plantas Tóxicas: Conhecimento de 
populares para prevenção de acidentes. Revista Intertox de Toxicologia, Risco Ambiental e Sociedade, v.7, n.2, p.17-36, 2014.

SIVIERO, A. et al.. Cultivo de Espécies Alimentares em Quintais Urbanos de Rio Branco, Acre, Brasil. Acta Botanica Brasilica. v.25, n.3, p.549-56, 2011. SOMAVILLA, N.; CANTO-DOROW, T.S. Levantamento das plantas medicinais utilizadas em bairros de Santa Maria, RS, Brasil. Ciência e Natura, v.18, p.131-48, 1996.

SOUZA, V.C.; LORENZI, H. Botânica Sistemática: guia ilustrado para identificação das famílias de Angiospermas da flora brasileira, baseado em APGII. São Paulo: Nova Odessa, Instituto Plantarum. 2005. 640p.

USTULIN, M. et al. Plantas medicinais comercializadas no Mercado Municipal de Campo Grande-MS. Revista Brasileira de Farmacognosia, v.19, n.3, p.805-13, 2009.

VEIGA-JÚNIOR, F.V. Estudo do consumo de plantas medicinais na Região Centro-Norte do Estado do Rio de Janeiro: aceitação pelos profissionais de saúde e modo de uso pela população. Revista Brasileira de Farmacognosia, v.18, n.2, p.30813, 2008.

VEIGA-JÚNIOR, V.F.V.; PINTO, A.C.; MACIEL, M.A.M. Plantas medicinais: cura segura? Química Nova, v.28, n.3, p.519-28, 2005.

VIGANÓ, J.; SILVA, C. Utilização de plantas medicinais pela população da região urbana de três barras do Paraná. Acta Scientiarum Health Science, v.29, n.1, p.51-8, 2007.

WORLD HEALTH ORGANIZATION (WHO). The world medicines situation 2011. Traditional medicines: global situation, issues and challenges. Geneva: WHO, 2011. 14p. 